\title{
HEMOGLOBIN-FACILITATED DIFFUSION OF OXYGEN: INTERFACIAL AND THICKNESS EFFECTS
}

\section{JOHN A. JACQUEZ, HOWARD KUTCHAI and EUGENE DANIELS}

\author{
Dept. of Physiology, The Medical School, Dept. of Biostatistics, School of Public Health, The University \\ of Michigan; McCollum-Pratt Institute, The Johns Hopkins University; Dept. of Biostatistics, School of \\ Public Health, The University of Michigant
}

\begin{abstract}
The equations of facilitated diffusion were solved numerically for steady state diffusion of oxygen across membranes of hemoglobin and myoglobin. An interfacial resistance was included in the boundary conditions and the dependence of the solutions on membrane thickness and interfacial conductance was studied. The data of Wittenberg on millipore membranes was fitted adequately if a tortuosity factor for the millipore membranes was taken into account. Comparison of the solutions with Wittenberg's data shows that the interfacial conductances must be large, $10^{-8} \mathrm{~mole} / \mathrm{cm}^{2}-\mathrm{sec}-\mathrm{mm} \mathrm{Hg}$ or larger. More accurate estimates of interfacial conductance could be obtained from data on thin membranes, $1-5 \mu$, but such data are not available. It was found that the concentration profiles are not independent of thickness and that the facilitation decreases as the membrane thickness decreases. The latter occurs even for zero interfacial resistance and hence cannot be attributed to a back pressure effect. The effect is present because the dissociation reaction at the low $\mathrm{P}_{\mathrm{O}_{2}}$ boundary increasingly becomes the limiting factor in the establishment of the steady state as $\mathrm{L}$ decreases.
\end{abstract}

$\begin{array}{ll}\text { Facilitated diffusion } & \begin{array}{l}\text { Myoglobin } \\ \text { Oxygen }\end{array} \\ \text { Membrane } & \end{array}$

Much work has been done recently on the solution of the equations for the facilitated diffusion of oxygen through membranes containing solutions of hemoglobin and myoglobin. Kreuzer (1970) and Wittenberg (1970) have reviewed this field in detail. Murray (1971) has obtained solutions using singular perturbation methods (Murray, $1968 \mathrm{a}, \mathrm{b})$. However, he retained only the zero'th order solution which implies local equilibrium throughout the membrane. As a consequence he can satisfy only two boundary conditions since this solution satisfies only the reduced set of differential equations. For boundary conditions Murray assumes that the dissolved oxygen at the high $\mathrm{P}_{\mathrm{O}_{2}}$ (oxygen partial pressure) boundary is in equilibrium with the adjacent gas phase and then chooses the value of the fraction of oxygenated protein on the low 
$\mathrm{P}_{\mathrm{O}_{2}}$ boundary so as to yield the measured facilitated oxygen flux. Since measurements of the facilitated component of the oxygen flux through hemoglobin solutions (Wittenberg, 1966) show an essentially inverse relationship with membrane thickness, Wittenberg (1970) with the aid of Murray's analysis concluded that the profiles of oxygen and oxyhemoglobin are independent of membrane thickness down to thicknesses of $1 \mu$ when plotted in terms of dimensionless variables. However, since the singular perturbation technique is applicable only to hemoglobin solutions which are thicker than roughly $10 \mu$ (Murray, 1971) and Murray's boundary conditions depend crucially on facilitated flux measurements, which are not available for very thin membranes, one must view Wittenberg's conclusion as being speculative.

Kreuzer and Hoofd (1970) have also found approximate solutions for the equations of facilitated diffusion. Although their derivation is not presented in the language of singular perturbation theory but is based on physical reasoning, their results can be derived in this context. An important difference between the method of Kreuzer and Hoofd and that of Murray is the treatment of the boundary layers in the membrane. Whereas Murray (1971) assumes local equilibrium exists all the way to the edges of the membrane, Kreuzer and Hoofd find separate approximate solutions for a thin boundary layer at each of the surfaces of the membrane. By this approach one finds lowest order corrections to the zero'th order perturbation solution. More importantly the Kreuzer and Hoofd method requires four boundary conditions for the complete solution since the order of the set of differential equations has not been reduced, although the condition that neither hemoglobin nor myoglobin can flow across the boundaries of the membrane is implicit in the linearized approximations for the boundary layer solution. For their calculations they set the oxygenated protein flux equal to zero at both boundaries and assume equilibrium of the oxygen between the gas and liquid phases at both edges of the membrane as boundary conditions. Since the boundary layer corrections vary inversely with membrane thickness for thick membranes, the Kreuzer-Hoofd solution approaches the same functional form as Murray's for thick membranes and differs from it only in the choice of a boundary value at the low $\mathrm{P}_{\mathrm{O}_{2}}$ side of the membrane. As with Murray's, their results are applicable to hemoglobin membranes that are greater than approximately $10 \mu$ in thickness.

In a paper by Kutchai et al. (1970) the non-linear differential equations were solved numerically using the method of quasilinearization (Jacquez, 1970). The boundary conditions used were the same type as those used by Kreuzer and Hoofd (1970) although the actual values differed. It was found that if the curves of the oxygen and oxyhemoglobin concentrations were plotted in terms of dimensionless variables that the concentration profiles changed with membrane thickness. However, Wittenberg (1970) criticized this work for the parameter values taken for the reaction rate constants and for use of the assumption of equilibrium of the oxygen between gas and liquid phases at the low $\mathrm{P}_{\mathrm{O}_{2}}$ boundary. The equilibrium assumption seemed reasonable because data (Wittenber, 1966) on the dependence of the oxygen flux on membrane thickness suggested that the resistance of the boundaries to the oxygen flux was 
negligible for membranes greater than $64 \mu$ in thickness. However, even a small interfacial resistance to oxygen flow could have a significant effect on the low $\mathrm{P}_{\mathrm{O}_{2}}$ boundary. In this way an interfacial resistance might add credence to Murray's local equilibrium solution (Murray, 1971) which implies a resistance at the low $\mathrm{P}_{\mathrm{O}_{2}}$ boundary.

For these reasons it seemed important to us to solve the equations of facilitated diffusion with the method of quasilinearization using improved parameters and boundary conditions which incorporate the possibility of interfacial resistance. In this paper we report the results of this study.

\section{Theory and methods}

\section{THE EQUATIONS OF STEADY STATE FACILITATED DIFFUSION}

Following the notation of Kutchai, Jacquez and Mather (1970), let w be the concentration of oxygen, let $\mathrm{u}$ be the concentration of free carrier $(\mathrm{Hb}$ or $\mathrm{Mb})$ and let $\mathrm{v}$ be concentration of carrier- $\mathrm{O}_{2}$ complex. The steady state equations are given by eqs. (1).

$$
\begin{aligned}
& D_{w} \frac{d^{2} w}{d x^{2}}=k_{1}(b-v) w-k_{2} v \\
& D_{v} \frac{d^{2} v}{d x^{2}}=-k_{1}(b-v) w+k_{2} v .
\end{aligned}
$$

In eqs. (1) $D_{w}$ and $D_{v}$ are the diffusion coefficients for $w$ and $v$ respectively; $u$ and $v$ are assumed to have the same diffusion coefficients, consequently the total carrier concentration is constant throughout the membrane, $u+v=b$. The constants $k_{1}$ and $k_{2}$ are the rate constants of the reaction (2).

$$
\mathrm{w}+\mathrm{u} \underset{\mathrm{k}_{2}}{\stackrel{\mathrm{k}_{1}}{\rightleftharpoons}} \mathrm{v} \text {. }
$$

Reaction (2) and thus eqs. (1) are exact for myoglobin but are approximations for hemoglobin. However this approximation has been used in all previous studies and the rationale for its use and its drawbacks have been discussed (Wittenberg, 1970; Kutchai et al., 1970).

\section{THE BOUNDARY CONDITIONS}

The membrane is of thickness $\mathrm{L}$, and we assume the partial pressure of oxygen is higher on the side for which $x=0$. Since the protein molecules cannot cross the interfaces, the flux of $v$ at the boundaries must be zero. Hence one set of boundary conditions is given by (3).

$$
\left.\frac{\mathrm{dy}}{\mathrm{dx}}\right|_{\mathbf{x}=0}=\left.\frac{\mathrm{dv}}{\mathrm{dx}}\right|_{\mathbf{x}=\mathbf{L}}=0 .
$$

Let $P(0)$ and $P(L)$ be the partial pressures of $\mathrm{O}_{2}$ just inside the membrane at the 
boundaries of the membrane and $\mathrm{P}_{0}$ and $\mathrm{P}_{\mathrm{L}}$ be the partial pressures in the gas phases just outside the membrane. In the studies of Kutchai $e t$ al. (1970) it was assumed that the interface presents no resistance to the flux of $\mathrm{O}_{2}$ so that $\mathrm{P}(0)=\mathrm{P}_{0}, \mathrm{P}(\mathrm{L})=\mathrm{P}_{\mathrm{L}}$ and hence $w(0)=\alpha_{s} P_{0}, w(L)=\alpha_{s} P_{L}$ where $\alpha_{s}$ is the solubility coefficient of the membrane solution for oxygen. As Wittenberg (1970) points out, this is probably a good assumption for the high $\mathrm{P}_{\mathrm{O}_{2}}$ boundary but may be poor at the low $\mathrm{P}_{\mathrm{O}_{2}}$ side. The concentration of oxyhemoglobin is quite sensitive to the partial pressure of oxygen at low partial pressures so that the shape of its concentration curve at the low $\mathrm{O}_{2}$ side of the membrane might change appreciably with small changes in $P(L)$. Thus even small values of interfacial resistance might have significant effects on the concentration profiles at the low oxygen side of the membrane.

In general the boundary conditions should include the possibility of a resistance to the $\mathrm{O}_{2}$ flux at the interfaces (Davies and Rideal, 1963). Such a resistance could be given by a monolayer of lipid or protein, a small unstirred boundary layer of gas or a combination of these. It should be noted that unless special precautions are taken it is difficult to prepare solutions which do not have some contaminating lipids in the interface. Furthermore hemoglobin itself forms monolayers at air-water interfaces (Guastalla, 1939). Assuming that the flux at the boundaries of the membrane is proportional to a drop in partial pressure across the interfaces we can account for a resistance at the interfaces with the boundary conditions given by eqs. (4).

$$
\begin{aligned}
& J_{t}=G\left[P_{0}-P(0)\right] \\
& J_{t}=G\left[P(L)-P_{L}\right] .
\end{aligned}
$$

Note that $G$ is a conductance and has units of flux $/ \mathrm{mm} \mathrm{Hg}$. For given $J_{\mathfrak{t}}$, if $\mathrm{G}$ is very large, $P_{0} \simeq P(0)$ and $P(L) \simeq P_{L}$. However, $J_{t}$ is not known and in fact should come out of the solution of the problem. Addition of eqs. (1) and integration gives relation (5) for the steady state total flux at any distance, $x$, in the membrane.

$$
J_{t}=-D_{w} \frac{d w}{d x}-D_{v} \frac{d v}{d x}
$$

It is a property of the steady state that $\mathrm{J}_{\mathrm{t}}$ is independent of $\mathrm{x}$ [see Fatt and LaForce (1961)]. If we substitute eq. (5), evaluated at the boundaries into eqs. (4) and set $\mathrm{P}(0)=\mathrm{w}(0) / \alpha_{\mathrm{s}}$ and $\mathrm{P}(\mathrm{L})=\mathrm{w}(\mathrm{L}) / \alpha_{\mathrm{s}}$, we obtain eqs. (6) and (7) which do not contain the unknown flux, $J_{t}$.

$$
\begin{aligned}
& \frac{w(0)}{\alpha_{s}}-\left.\frac{D_{w}}{G} \frac{d w}{d x}\right|_{0}=P_{0} \\
& \frac{w(L)}{\alpha_{s}}+\left.\frac{D_{w}}{G} \frac{d w}{d x}\right|_{L}=P_{L} .
\end{aligned}
$$

Boundary conditions of this type are generally known as mixed boundary conditions. Equations (3), (6) and (7) give exact boundary conditions and in theory eqs. (1) are 
solvable if we know the parameters $k_{1}, k_{2}, \alpha_{s}, D_{w}, D_{v}, G$ and $b$ and the partial pressures in the gas phases, $\mathrm{P}_{0}$ and $\mathrm{P}_{\mathrm{L}}$.

Although eqs. (6) and (7) provide us with exact boundary conditions there is still some question in practice about the true value of $\mathrm{P}_{\mathrm{L}}$. On the high $\mathrm{P}_{\mathrm{O}_{2}}$ side there is no problem. But in experiments the low $\mathrm{P}_{\mathrm{O}_{2}}$ side is usually flushed with $\mathrm{O}_{2}$-free gas and because a small amount of $\mathrm{O}_{2}$ is constantly entering this gas phase from the membrane $P_{L}$ cannot be zero. For example, using the data in fig. 1 of Wittenberg's paper (Wittenberg, 1966) one finds that for a $150 \mu$ membrane with $P_{0}=200 \mathrm{~mm} \mathrm{Hg}$ the sxygen flux is $3.5 \mu \mathrm{l} / \mathrm{min}$. A stream of helium flowing at the rate of $60 \mathrm{ml} / \mathrm{min}$ was used to flush the chamber on the low oxygen side of the membrane. Since the total pressure was maintained a $1 \mathrm{~atm}$, the assumption of uniform mixing of the oxygen flux with the flushing stream would imply that $\mathrm{P}_{\mathrm{L}}$ was $0.044 \mathrm{~mm} \mathrm{Hg}$. For a $25 \mu$ membrane exposed to the same high side $\mathrm{P}_{\mathrm{O}_{2}}$ the oxygen flux would be about 6 fold greater than for the $150 \mu$ membrane. Thus for the same flushing rate $\mathrm{P}_{\mathrm{L}}$ would be about $0.25 \mathrm{~mm} \mathrm{Hg}$. However, in Wittenberg's experiments the helium stream was directed at the surface of the membrane and we are not certain that the uniform mixing assumption is valid. Lacking more information we have arbitrarily set $P_{L}=0.05 \mathrm{~mm} \mathrm{Hg}$ for all of our computations. In the Appendix it is demonstrated that small changes in $P_{L}$ will make negligible difference in the results if the surface conductance, $\mathrm{G}$, is $10^{-9}$ or less. Actually, if provision is made to obtain uniform mixing in the chamber on the low $\mathrm{P}_{\mathrm{O}_{z}}$ side, the dependence of $P_{L}$ on the flux, $J_{t}$, and the flushing rate can be incorporated into the boundary conditions. Let $\mathrm{V}$ be the volume flow rate at STP in $\mathrm{ml} / \mathrm{sec}$ of the flushing gas, let $\mathrm{J}_{\mathrm{t}}$ be the $\mathrm{O}_{2}$ flux in moles $/ \mathrm{cm}^{2} / \mathrm{sec}$, and let $\mathrm{A}$ be the surface area of the membrane in $\mathrm{cm}^{2}$. Then, if we assume the flushing gas is oxygen free, the flushing rate is much greater than the oxygen flux and the total pressure is $760 \mathrm{~mm} \mathrm{Hg}, \mathrm{P}_{\mathrm{L}}$ is given by eq. (8).

$$
P_{L}=\frac{A}{V} J_{t}\left(22.4 \times 10^{3}\right)(760) \mathrm{mm} \mathrm{Hg}
$$

Substituting for $P_{L}$ in the second of eqs. (4) gives eq. (9) to replace eq. (7).

$$
\frac{w(L)}{\alpha_{s}\left[1+17 \times 10^{6} A G / V\right]}+\left.\frac{D_{w}}{G} \frac{d w}{d x}\right|_{L}=0 \text {. }
$$

We have used eqs. (6) and (7) but eq. (9) could be used instead of (7) if provision is made to obtain uniform, rapid mixing of gases in the chamber on the low oxygen side of the membrane.

\section{INTERFACLAL $\mathrm{O}_{2}$ CONDUCTIVITY}

We must decide what numerical values to use for G. Wittenberg (1966) found that the flux of $\mathrm{N}_{2}$ and the facilitated flux of $\mathrm{O}_{2}$ across millipore filters filled with hemoglobin solution was inversely proportional to thickness for filters from $64 \mu$ to $300 \mu$ thick. The proportionality may extend down to $25 \mu$ thickness as well. If the $\mathrm{O}_{2}$ flux resis- 
tance of the interface $(1 / \mathrm{G})$ is taken to be constant, analogy with electrical resistances shows that the effect of the interfaces should become greater with decreasing layer thickness. The proportionality observed by Wittenberg shows that the $\mathrm{O}_{2}$ pressure drops across the interfaces are negligible at least in layers thicker than $64 \mu$ and possibly in layers $25 \mu$ and thicker. In layers thinner than this, the interfacial resistance may play an important role.

To obtain a rough estimate of a lower bound for $\mathrm{G}$ consider the following. If we treat the interface as though it were a layer of water of thickness $\varepsilon$, the $\mathrm{O}_{2}$ flux across the interface can be written,

$$
\mathrm{J}_{\mathrm{t}}=\mathrm{G} \Delta \mathrm{P}=\frac{\mathrm{D}_{\mathrm{w}} \alpha_{\mathrm{s}}}{\varepsilon} \Delta \mathrm{P}
$$

so that $G=D_{w} \alpha_{s} / \varepsilon$. The precision of Wittenberg's results seems to rule out the effective value of $\varepsilon$ being more than $10 \mu$. Taking $\mathrm{D}_{\mathrm{w}}=1.7 \times 10^{-5} \mathrm{~cm}^{2} / \mathrm{sec}, \alpha_{\mathrm{s}}=1.5 \times$ $10^{-9} \mathrm{~mole} /(\mathrm{ml}-\mathrm{mm} \mathrm{Hg})$, and $\varepsilon=10^{-3} \mathrm{~cm}$ we compute $\mathrm{G}=2.5 \times 10^{-11} \mathrm{~mole} /\left(\mathrm{cm}^{2}\right.$ sec-mm $\mathrm{Hg}$ ) as a lower limit for $\mathrm{G}$. It might be noted tuat Murray's solutions imply a value of $\mathrm{G}$ in the range of $10^{-10}$ to $10^{-9}$. We have solved the problem for $\mathrm{G}=10^{-11}$, $10^{-10}, 10^{-9}, 10^{-8}$ and for $\mathrm{G}=\infty$ for various values of $\mathrm{L}$. We know of no experimentally determined values for $G$ for the air-water interface.

\section{THE NORMALIZED EQUATIONS}

As in our previous paper (Kutchai, Jacquez and Mather, 1970), we normalize eqs. (1) with use of the following transformations:

$$
\begin{aligned}
\mathrm{W} & =\mathrm{w} / \mathrm{w}_{\mathrm{o}} \\
\mathrm{V} & =\mathrm{v} / \mathrm{b} \\
\mathrm{y} & =\mathrm{x} / \mathrm{L} .
\end{aligned}
$$

In eqs. (11), $b$ is total carrier concentration, $b=u+v$ and $w_{0}$ is defined to be $\alpha_{s} P_{0}$, i.e. it is the hypothetical concentration for solution in equilibrium with the $\mathrm{P}_{\mathrm{O}_{2}}$ at the high side. With these transformations eqs. (1) become:

$$
\begin{aligned}
& 0=\frac{\mathrm{d}^{2} \mathrm{~V}}{\mathrm{dy}^{2}}+\alpha \gamma(1-\mathrm{V}) \mathrm{W}-\alpha \mathrm{V} \\
& 0=\frac{\mathrm{d}^{2} \mathrm{~W}}{\mathrm{dy}^{2}}-\alpha \beta \gamma(1-\mathrm{V}) \mathrm{W}+\alpha \beta \mathrm{V}
\end{aligned}
$$

where the dimensionless parameters in eqs. (12) are as follows:

$$
\begin{aligned}
& \alpha=\mathrm{k}_{2} \mathrm{~L}^{2} / \mathrm{D}_{\mathrm{v}} \\
& \beta=\mathrm{D}_{\mathrm{v}} \mathrm{b} / \mathrm{D}_{\mathbf{w}} \mathrm{w}_{0} \\
& \gamma=\mathrm{k}_{1} \mathrm{w}_{0} / \mathbf{k}_{2} .
\end{aligned}
$$


In terms of the normalized variables the boundary conditions are given by eqs. (14).

$$
\begin{aligned}
& \left.\frac{\mathrm{dV}}{\mathrm{dy}}\right|_{y=0}=0 \\
& \left.\frac{\mathrm{dV}}{\mathrm{dy}}\right|_{y=1}=0 \\
& W(0)-\left.\frac{\alpha_{s} D_{w}}{\mathrm{GL}} \frac{\mathrm{dW}}{\mathrm{dy}}\right|_{y=0}=1 \\
& W(1)+\left.\frac{\alpha_{s} D_{w}}{\mathrm{GL}} \frac{\mathrm{dW}}{\mathrm{dy}}\right|_{y=1}=\frac{P_{L}}{P_{0}} .
\end{aligned}
$$

\section{NUMERICAL METHODS AND COMPUTATIONS}

Equations (12) with boundary conditions (14) were solved by the method of quasilinearization (Jacquez, 1970). The method was programmed in FORTRAN IV and the computations were carried out in double precision arithmetic on the IBM 360/67 at the University of Michigan Computing Center. A finite difference approach is used in which the membrane is divided into $2^{\mathrm{r}}$ equal intervals. Initially, results were computed using 16 intervals. The number of intervals were than increased until successive computations yielded results which were identical to five significant digits; 64 intervals were used for all of the computations reported in this paper. The user initiates the process by providing an initial guess for the solution and the program then iteratively generates a sequence of approximations. If the initial guess is not close enough to the true solution the process does not converge but if it converges it converges quadratically. For each value of $G$ the initial trial solution which we used for $\mathrm{L}=1 \mu$ was a linear gradient across the membrane in $\mathrm{W}(\mathrm{y})$ and a $\mathrm{V}(\mathrm{y})$ which was in equilibrium with $\mathrm{W}(0)$. This converged for all cases. This solution when used as the initial guess for $\mathrm{L}=2 \mu$ gave convergence; the latter solution was then used as the initial guess to obtain the solution for $\mathrm{L}=5 \mu$. In this way we successively generated solutions for 1 to $300 \mu$ membranes with interfacial conductances $\mathrm{G}$, of $10^{-11}, 10^{-10}$, $10^{-9}, 10^{-8}$ and $\infty$.

\section{Results and discussion}

\section{RESULTS}

Computations were first carried out with use of the parameters for $15 \%$ human hemoglobin for membranes of thickness $1,2,5,10,25,50,100,150$ and $300 \mu$ and for surface conductances, $\mathrm{G}=\infty, 10^{-8}, 10^{-9}, 10^{-10}, 10^{-11} \mathrm{moles} / \mathrm{sec} \mathrm{cm}^{2} \mathrm{~mm} \mathrm{Hg}$. Partial pressures of oxygen of 200 and $0.05 \mathrm{~mm} \mathrm{Hg}$ were chosen for the high $\left(\mathrm{P}_{0}\right)$ and low $\left(\mathrm{P}_{\mathrm{L}}\right)$ sides, respectively. Table 1 gives the values of the parameters used and the reference from which the value of the parameter was obtained. In the study of Kutchai et al. (1970) and in the computations of this study a value of $1.8 \times 10^{-9} \mathrm{~mole} / \mathrm{ml}-\mathrm{mm}$ 

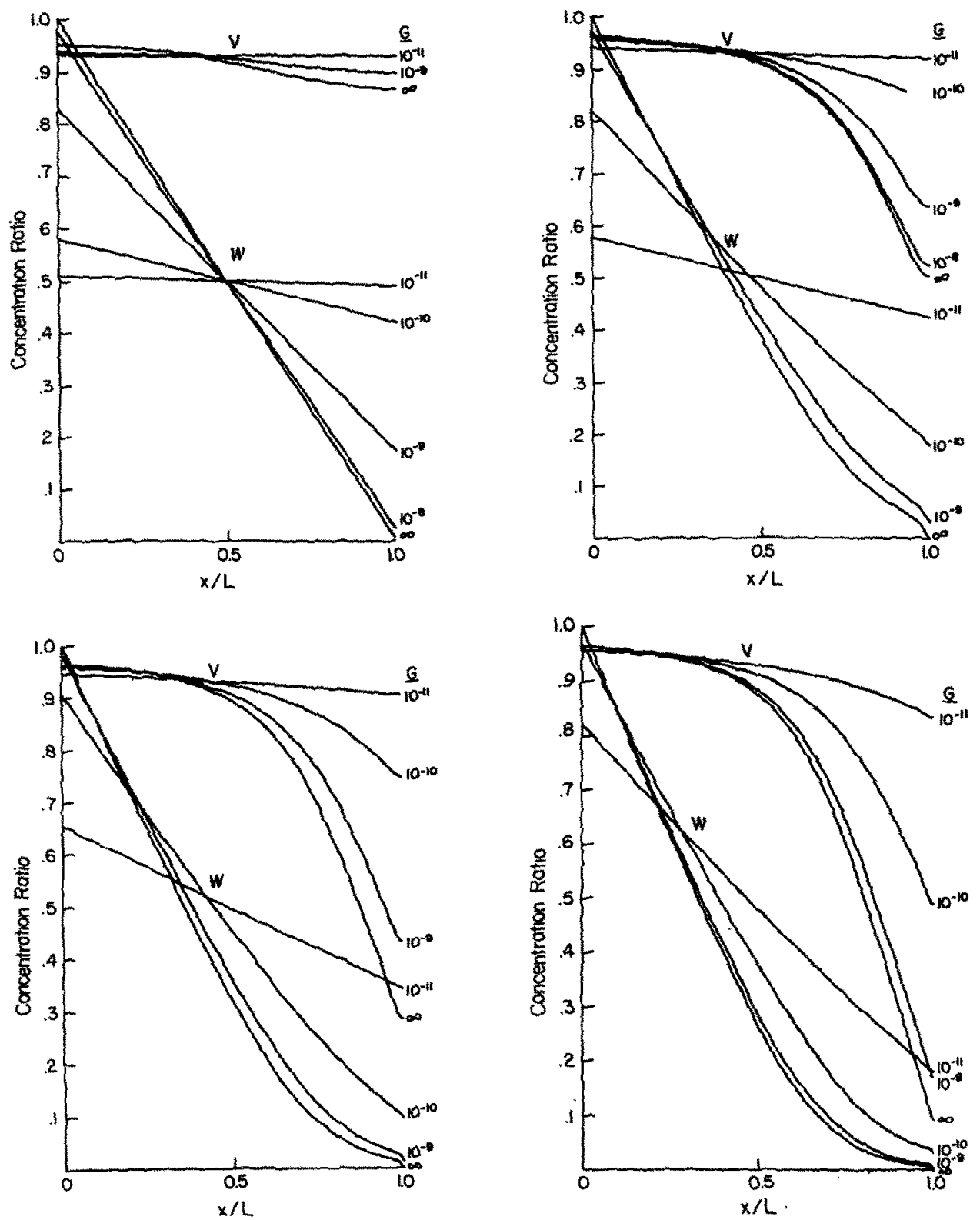

Fig. 1. Computed concentration profiles for the facilitated diffusion of oxygen through $15 \%$ hemoglobin for interfacial conductances of $10^{-11}$ to $\infty$ and for membranes of thickness $1,10,25,100 \mu$.

$\mathrm{Hg}$ was used for the solubility of oxygen in hemoglobin solutions. Kreuzer and Hoofd (1970) give a value of $1.663 \times 10^{-9}$. For his computations on the time course of uptake of $\mathrm{O}_{2}$ by thin layers of $34 \%$ hemoglobin, Kutchai $(1970)$ used the value reported by Sendroy, Dillon and Van Slyke (1934) for red cells, $1.51 \times 10^{-9} \mathrm{~mole} / \mathrm{ml}-\mathrm{mm} \mathrm{Hg}$. We 
TABLE 1

Values of parameters used for hemoglobin and myoglobin

\begin{tabular}{|c|c|c|c|c|}
\hline Parameter & Units & $15 \%$ & $34 \%$ & $\begin{array}{l}\text { Horse myoglobin } \\
11.5 \%\end{array}$ \\
\hline b & $\mathrm{mole} / \mathrm{cm}^{3}$ & $0.9375 \times 10^{-5}$ & $2.125 \times 10^{-5}$ & $\begin{array}{l}7.2 \times 10^{-6} \\
\text { (Wittenberg, 1966) }\end{array}$ \\
\hline$D_{v}$ & $\mathrm{~cm}^{2} / \mathrm{sec}$ & $3.6 \times 10^{-7}$ & $6.8 \times 10^{-8}$ & $\begin{array}{l}8.6 \times 10^{-7} \\
\text { (Wittenberg, 1970) }\end{array}$ \\
\hline$D_{w}$ & $\mathrm{~cm}^{2} / \mathrm{sec}$ & $1.4 \times 10^{-5}$ & $7.0 \times 10^{-6}$ & $\begin{array}{l}1.5 \times 10^{-5} \\
\text { (Kreuzer and Hoofd, 1970) }\end{array}$ \\
\hline $\mathrm{k}_{1}$ & $\mathrm{~cm}^{3} /$ mole-sec & $3.0 \times 10^{9}$ & $3.0 \times 10^{9}$ & $\begin{array}{l}14.0 \times 10^{9} \\
\text { (Wittenberg, 1966) }\end{array}$ \\
\hline $\mathbf{k}_{2}$ & $\sec ^{-1}$ & 40 & 40 & $\begin{array}{l}11 \\
\text { (Wittenberg, 1966) }\end{array}$ \\
\hline$\alpha_{\mathrm{s}}$ & $\mathrm{mole} / \mathrm{cm}^{3} \mathrm{~mm} \mathrm{Hg}$ & $\begin{array}{l}1.8 \times 10^{-9} \\
\text { (Kutchai } \text { et } \\
\text { al., 1970) }\end{array}$ & $\begin{array}{l}1.8 \times 10^{-9} \\
\text { (Kutchai et al., } \\
1970)\end{array}$ & $\begin{array}{l}1.5 \times 10^{-9} \\
\text { (Kutchai, 1970) }\end{array}$ \\
\hline
\end{tabular}

TABLE 2

Summary of findings for $15 \%$ hemoglobin, $G=\infty . P_{0}=200 \mathrm{~mm} \mathrm{Hg} . P_{L}=0.05 \mathrm{~mm} \mathrm{Hg}$.

Membrane thickness-microns

\begin{tabular}{|c|c|c|c|c|c|c|c|c|c|}
\hline & 1 & 2 & 5 & 10 & 25 & 50 & 100 & 150 & 300 \\
\hline$V(0)$ & 0.9523 & 0.9597 & 0.9627 & 0.9635 & 0.9639 & 0.9641 & 0.9642 & 0.9642 & 0.9643 \\
\hline$V(1)$ & 0.8678 & 0.8013 & 0.6594 & 0.5018 & 0.2858 & 0.1673 & 0.0930 & 0.0654 & 0.0362 \\
\hline $\mathrm{V}(0) / \mathrm{V}_{\text {eq }}(0)^{*}$ & 0.9876 & 0.9953 & 0.9983 & 0.9991 & 0.9996 & 0.9998 & 0.9999 & 0.9999 & 1.0000 \\
\hline $\mathrm{V}(1) / \mathrm{V}_{\mathrm{eq}}(1)^{*}$ & 129.4 & 119.5 & 98.35 & 74.84 & 42.63 & 24.96 & 13.876 & 9.759 & 5.406 \\
\hline $\mathrm{F}$ (facilitation) ${ }^{* *}$ & 0.0566 & 0.1061 & 0.2031 & 0.3092 & 0.4543 & 0.5335 & 0.5834 & 0.6020 & 0.6214 \\
\hline $10^{10} \cdot J_{F}\left(\frac{\text { moles }}{\mathrm{cm}^{2}-\mathrm{sec}}\right)^{\dagger}$ & 28.5 & 26.7 & 20.5 & 15.6 & 9.15 & 5.38 & 2.94 & 2.02 & 1.04 \\
\hline $10^{10} \cdot \mathrm{J}_{\mathrm{D}}\left(\frac{\text { moles }}{\mathrm{cm}^{2}-\mathrm{sec}}\right)$ & 504 & 252 & 101 & 50.4 & 20.2 & 10.1 & 5.04 & 3.36 & 1.68 \\
\hline$f_{\mathrm{F}}\left(\frac{\mu \mathrm{l}}{\mathrm{cm}^{2}-\mathrm{min}}\right)$ & 3.84 & 3.59 & 2.75 & 2.09 & 1.23 & 0.723 & 0.395 & 0.272 & 0.140 \\
\hline
\end{tabular}

* $V_{\text {eq }}(0)$ and $V_{\text {eq }}(1)$ are calculated equilibrium values for $V$ for the $W(0)$ and $W(1)$ present $W(0)=1, W(1)=0.00025$, $V_{c q}(0)=0.9643, V_{\text {eq }}(1)=0.0067$ for all membrane thicknesses.

** The facilitation $F$ is defined as $J_{F} / J_{D}$.

${ }^{\dagger} J_{F}$ and $J_{D}$ are the facilitated and diffusive fuxes respectively.

$+\oiint_{\mathrm{F}}$ is the facilitated flux in $\mu 1 / \mathrm{cm}^{2}-\mathrm{min}$. A is the area for a millipore filter used by Wittenberg. i.e. $9.09 \mathrm{~cm}^{2}$. 
TABLE 3

Summary of findings for $11.5 \%$ Myoglobin $G=\infty . P_{0}=200 \mathrm{~mm} \mathrm{Hg} . P_{L}=0.05 \mathrm{~mm} \mathrm{Hg}$. Membrane thickness-microns

\begin{tabular}{lccll}
\hline $\mathrm{V}(0)$ & 0.9967 & 0.9974 & 0.9974 & 0.9974 \\
$\mathrm{~V}(1)$ & 0.9878 & 0.9383 & 0.8495 & 0.5432 \\
$\mathrm{~V}(0) / \mathrm{V}_{\text {eq }}(0)^{*}$ & 0.9993 & 1.0000 & 1.0000 & 1.0000 \\
$\mathrm{~V}(1) / \mathrm{V}_{\text {eq }}(1)^{*}$ & 11.34 & 10.77 & 9.75 & 6.23 \\
$\mathrm{~F}$ & 0.01225 & 0.0813 & 0.2036 & 0.6251 \\
$10^{10} \mathrm{~J}_{\mathbf{F}}$ & 5.51 & 3.66 & 3.66 & 2.81 \\
$10^{10} \mathrm{~J}_{\mathrm{D}}$ & 450 & 45.0 & 18.0 & 4.50 \\
$\mathscr{J}_{\mathrm{F}}$ & 0.741 & 0.492 & 0.492 & 0.378 \\
\hline
\end{tabular}

* For these membranes, $W(0)=1.0000, W(1)=0.00025, V_{\text {eq }}(0)=0.9974, V_{\mathrm{eq}}(1)=0.08714$

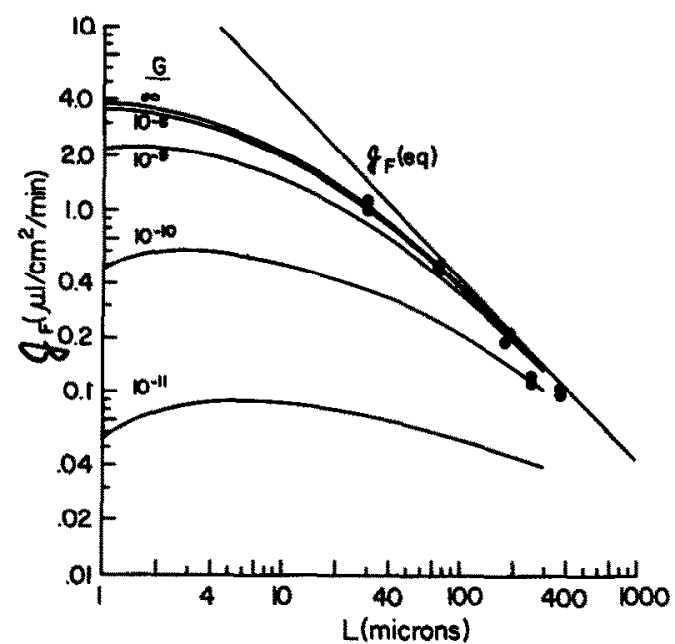

Fig. 2. Dependence of facilitated flux on membrane thickness and interfacial conductance for $15 \%$ hemoglobin. Wittenberg's results corrected for a tortuosity factor of 1.44 and for the difference in hemoglobin concentrations are shown by the points-

have not repeated our computations with these other values for $\alpha_{\mathrm{s}}$ because the results cannot differ much and the extra cost is considerable. Rather than repeat the computations for many combinations of the parameters and partial pressures we would prefer to wait and compute results for simulations of specific experiments for which the parameter values and experimental conditions are specified. However, it should be noted that the solubility of oxygen appears only in the boundary conditions, eqs. (6) and (7), not in the differential equations, eqs. (1), and it appears in the boundary conditions as a result of the relations $w(0)=P(0) \alpha_{s}, w(L)=P(L) \alpha_{s}$. If we divide eqs. (6) and (7) by $P_{0}$ then it may be seen that provided $b, k_{1}, k_{2}, D_{v}$ and $D_{w}$ are fixed, 


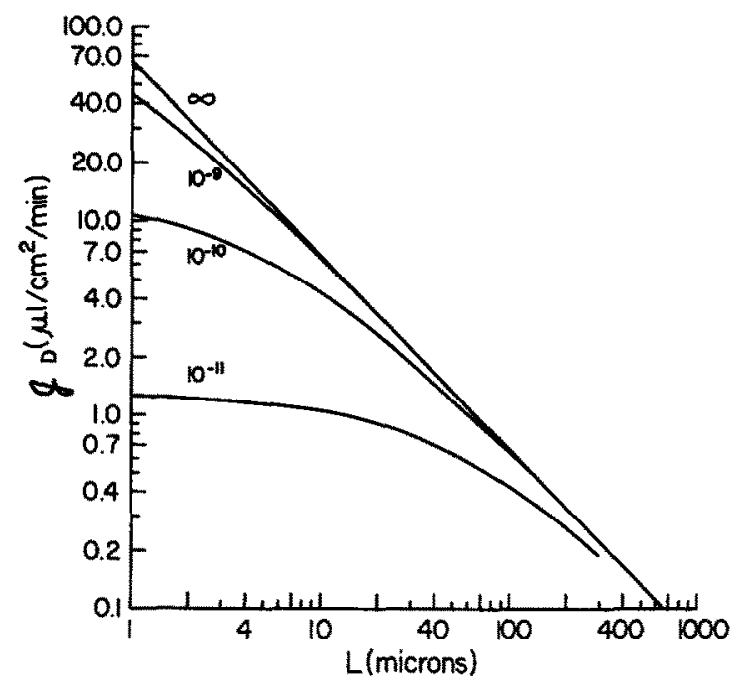

Fig. 3. Dependence of diffusive flux on membrane thickness and interfacial conductance for $15 \%$ hemoglobin.

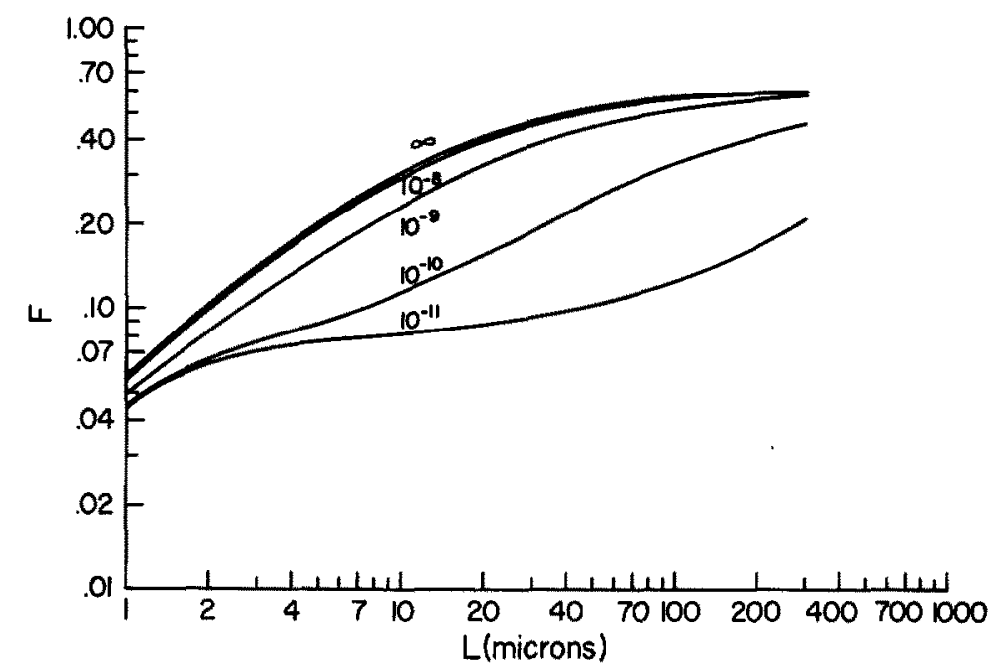

Fig. 4. Dependence of facilitation on membrane thickness and interfacial conductance for $15 \%$ hemoglobin.

the boundary conditions remain invariant if the combinations of parameters $\alpha_{\mathrm{s}} \mathrm{P}_{0}$, $\mathrm{GP}_{0}$ and $\mathrm{P}_{\mathrm{L}} / \mathrm{P}_{0}$ remain invariant.

Computations for $11.5 \%$ horse myoglobin were done for $L$ equal to $1,10,25$ and $100 \mu$ for the same range of values for $G$ as was used for the hemoglobin studies; $\alpha_{s}$ was taken as $1.5 \times 10^{-9}$ for the myoglobin computations. Figure 1 shows the concentration profiles obtained for $15 \%$ hemoglobin. We have also done the computations for $34 \%$ human hemoglobin for different membrane thicknesses. However, the graphs for $34 \%$ hemoglobin and $11.5 \%$ myoglobin are qualitatively similar to those for $15 \%$ 
hemoglobin and are not shown. Tables 2 and 3 summarize the results obtained for $15 \%$ hemoglobin and $11.5 \%$ myoglobin for $\mathrm{G}=\infty$. Summaries for other values of $\mathrm{G}$ are not given because our findings imply that $\mathrm{G}$ is $10^{-8}$ or larger in value and the results obtained for $G=10^{-8}$ differed little from those obtained for $G=\infty$. Figures 2 and 3 show the dependence of facilitated and diffusive fluxes on thickness and fig. 4 shows the facilitation, $F=J_{F} / J_{D}$, as a function of membrane thickness for $15 \%$ hemoglobin.

\section{COMPARISON WITH KREUZER-HOOFD FOR $\mathrm{G}=\infty$}

When $G$ is infinite our boundary conditions become the same as those used by Kreuzer and Hoofd (1970), and in view of the discussion in the introduction we should expect agreement between their results and ours for sufficiently thick membranes. We have computed the Kreuzer-Hoofd solutions using our parameters for $15 \%$ hemoglobin. Table 4 gives the difference between our result and that given by the

TABLE 4

Comparison of $\mathrm{K}$ reuzer-Hoofd results for $15 \%$ hemoglobin with ours

\begin{tabular}{rll}
\hline L & $\begin{array}{l}\text { Percent differences } \\
\text { in } \mathrm{J}_{\mathrm{t}}^{*}\end{array}$ & $\begin{array}{l}\text { Percent difference } \\
\text { in } \mathrm{J}_{\mathrm{F}}\end{array}$ \\
\hline$\mu$ & & \\
300 & 0.06 & 0.16 \\
100 & 0.06 & 0.17 \\
25 & 0.21 & 0.68 \\
10 & 1.16 & 4.92 \\
5 & 4.10 & 24.3 \\
\hline
\end{tabular}

* Difference between Kreuzer-Hoofd results and ours as \% of ours.

Kreuzer-Hoofd method, as percent of our result, for different membrane thicknesses. The total flux was obtained by integrating eq. (5) to obtain eq. (15), the first term of which

$$
J_{t}=\frac{D_{w} w_{0}}{L}[W(0)-W(1)]+\frac{D_{v} b}{L}[V(0)-V(1)]
$$

is the diffusive flux, $J_{D}$, and the second term the facilitated flux, $J_{F}$. The values of $V(0)$ and $V(1)$ given by the two methods were then substituted in the equation, the values of $\mathrm{W}(0)$ and $\mathrm{W}(1)$ being the same for the two methods. Thus the diffusive flux is calculated exactly by both methods, given $W(0)$ and $W(1)$ and the difference is in the calculated facilitated fluxes. However since the total flux is the experimentally measured quantity we give the percent deviation in that as well as in the calculated facilitated flux. Note how close the results are for membranes down to $25 \mu$ in thickness. Even for a $10 \mu$ membrane the deviation is not large but it increases markedly as one goes to thinner membranes. 


\section{EFFECTS OF MEMBRANE THICKNESS AND INTERFACIAL RESISTANCE}

It may be seen from figs. 1a-1d that for a given interfacial conductance the concentration profiles, plotted in dimensionless variables, are not independent of the membrane thickness. This effect is also present in the Kreuzer-Hoofd solutions. As a result $V(0)-V(1)$ in eq. (1) is not constant for fixed $W(0)$ and $W(1)$ so that the facilitated flux does not depend solely on $1 / \mathrm{L}$ as does the diffusive flux for fixed $W(0)$ and $W(1)$. If $V(0)-V(1)$ were constant for $G=\infty$ the facilitation, $F=J_{D} / J_{F}$, would be independent of membrane thickness but in fact both our results and those of Kreuzer and Hoofd predict that it must eventually decrease as membrane thickness decreases. Kutchai (1970) reaches the same conclusion from his studies of the time dependent equations for $\mathrm{O}_{2}$ uptake by layers of hemoglobin.

For thick membranes our results approach those of Kreuzer and Hoofd (1970). It should be noted that as $\mathrm{L} \rightarrow \infty$ the effect of any finite interfacial resistance eventually becomes negligible and hence for sufficiently large $L$ the dimensionless profiles become essentially invariant and $J_{F}$ becomes inversely related to membrane thickness. As shown in fig. 2 the smaller the interfacial conductance, $G$, the greater the thickness required to give an approximately $1 / \mathrm{L}$ behavior in $J_{F}$. This departure of $J_{F}$ from $1 / L$ behavior with decreasing $G$ is much more marked than for the diffusive flux, $J_{D}$, as is seen in fig. 3. The straight line in fig. 2 is obtained by replacing $V(0)$ and $V(1)$ in the equation for $\mathrm{J}_{\mathrm{F}}$ by $\mathrm{V}_{\mathrm{eq}}^{\infty}(0)$ and $\mathrm{V}_{\mathrm{eq}}^{\infty}(1)$, respectively, which are the values that $\mathrm{V}$ would take at the boundaries if the hemoglobin were in equilibrium with $W$ for the $G=\infty$ case. Thus the straight line is the large $L$ a symptote of $J_{F}$.

Wittenberg's data on the facilitated diffusion of oxygen through hemoglobin indicates that the facilitated flux is inversely proportional to membrane thickness down to $\mathrm{L}=64 \mu$ with possibly a small deviation for $\mathrm{L}=25 \mu$. We must conclude, therefore, that $\mathrm{G}$ must be of order $10^{-8}$ or greater.

In fig. 4 we have plotted the facilitation, $F=J_{F} / J_{D}$, as a function of membrane thickness for different values of $G$. For all values of $G, F$ approaches a fixed asymptotic value as $L$ increases, the approach to the asymptotic value being more rapid in $L$ for high values of $G$. The asymptotic value for large $L$ corresponds to the solution for which the oxygen-hemoglobin reaction may be assumed to be at equilibrium.

\section{COMPARISON WITH MURRAY'S SOLUTION}

It is not possible to compare our results with those of Murray in the same way as we have done in comparing them with those of Kreuzer and Hoofd. Murray's solution differs from the Kreuzer-Hoofd solution in that the reaction equilibrium assumption is extended to both boundaries and this reduces the number of boundary conditions by two. Whereas in our method and in that of Kreuzer and Hoofd the fluxes are determined by the solutions, Murray introduces the experimentally determined flux, $\mathrm{J}_{\mathrm{F}}$, into the problem and uses it to fix $\mathrm{V}(0)-\mathrm{V}(1)$. But as a result of this manuever the assumption of reaction equilibrium at the low $\mathrm{P}_{\mathrm{O}_{2}}$ boundary gives a value of $\mathrm{W}(1)$ which is too large for the experimental conditions which he simulates unless one 
assumes a value of $\mathrm{G}$ that falls between $10^{-9}$ and $10^{-10}$. But as we have shown (fig. 2) this pushes the region of $1 / \mathrm{L}$ behavior for the facilitated flux outside the range in which Wittenberg's results (1966) place it. Murray's solution can only be good for very thick membranes for which the reaction equilibrium assumption is good right out to the boundary and we have to conclude that it is really not adequate to describe the results Wittenberg obtained for his thinner $(25 \mu$ and $64 \mu)$ membranes.

\section{THE EFFECT OF TORTUOSITY}

To compare our results with Wittenberg's data we must take into account a correction for tortuosity of the channels in the millipore filters. Our calculated fluxes were usually 1.4-1.6 fold greater than Wittenberg's measured fluxes for a given thickness of membrane. The tortuosity effect has been discussed by Keller and Friedlander (1966) who found a factor of 1.63 for diffusion of methemoglobin through millipore filters and by Wittenberg (1970). Murray (1971) corrected for this by assuming the diffusion path was $220 \mu$ for a $150 \mu$ thick membrane, a factor of 1.47 . Kreuzer and Hoofd (1970) on the other hand used an L of $180 \mu$ in their calculations for comparison with Wittenberg's results on $150 \mu$ membranes. We have found that we need a correction factor in the range 1.40-1.50. The correction factor involved has to do with the estimation of the ratio of area for diffusion divided by path length, A/L. Recall that the measured amount of oxygen crossing a membrane is the membrane area multiplied by flux, $J_{t}$, of eq. (15). Thus the measured amount crossing a membrane is proportional to $(\mathrm{A} / \mathrm{L})$ and so we are faced with the problem of estimating this ratio if channels are not straight through and uniform in cross-section. The tortuosity correction is a correction from the measured ratio $(A / L)$ to an effective ratio $\left(A^{*} / L^{*}\right)$. For the diffusive flux the entire correction for non-straight through channels is in this ratio; for facilitated flux this is probably the main effect although we cannot exclude a further effect due to effective path length, $L^{*}$, because of the non-linear reaction terms. For specific geometries the correction in A/L may actually be partitioned between the estimated area and channel length. For example if the channels are all of uniform cross section of total area $A^{*}$ and channel length $L^{*}>L$ then the true ratio

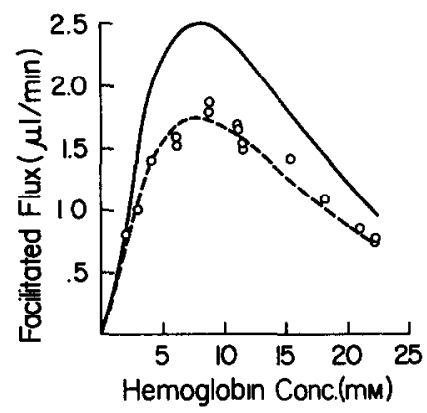

Fig. 5. Dependence of facilitated flux on hemoglobin concentration for a membrane of $150 \mu$ thickness and for $G=\infty$. Wittenberg's data $(O)$; our computed results $(-)$; computed result corrected for tortuosity factor of $1.44(--)$. 
is $\mathrm{A}^{*} / \mathrm{L}^{*}$. The measured membrane thickness is $\mathrm{L}$. If as is frequently done the cross sectional area is calculated from the measured volume entrained in the filter, $\mathrm{V}=\mathrm{AL}=$ $A^{*} L^{*}$, and $A / L=\left(A^{*} / L^{*}\right)\left(L^{*} / L\right)^{2}$. Thus for this geometry the measured ratio $A / L$ is $\left(\mathrm{L}^{*} / \mathrm{L}\right)^{2}$ times the true ratio $\left(\mathrm{A}^{*} / \mathrm{L}^{*}\right)$. We have corrected our results as though the correction could be partitioned between the area and channel length in this way. Thus points in fig. 2 are Wittenberg's data for $17.7 \%$ hemoglobin corrected for tortuosity by the factor $\left(L^{*} / L\right)^{2}=1.44$ and reduced to $15 \%$ hemoglobin by the approximate correction factor, $\left(D_{v} \cdot b\right)_{15 \%} /\left(D_{v} \cdot b\right)_{17.7 \%}=1.096$. Using the tortuosity factor correction of 1.44 we have also fitted Wittenberg's (1966) data which show the dependence of facilitated flux on hemoglobin concentration for a $150 \mu$ membrane. Figure 5 shows Wittenberg's data, our calculated results for a $150 \mu$ membrane and our results corrected for a tortuosity factor of 1.44.

\section{Appendix}

Referring to eq. (15) of the text, the facilitation is given by eq. (A1).

$$
F=\frac{D_{v}[v(0)-v(L)]}{D_{w}[w(0)-w(L)]}
$$

Hence the total flux can be written as in eq. (A2).

$$
J_{t}=(1+F) \frac{D_{w}}{L}[w(0)-w(L)]
$$

Substituting $w(0)=\alpha_{s} P(0)$ and $w(L)=\alpha_{s} P(L)$ gives eq. (A3).

$$
\mathrm{J}_{\mathrm{t}}=(1+\mathrm{F}) \frac{\alpha_{\mathrm{s}} \mathrm{D}_{\mathrm{w}}}{\mathrm{L}}[\mathrm{P}(0)-\mathrm{P}(\mathrm{L})]
$$

Add eq. (4) of the text to give the following eq. (A4).

$$
\frac{2 J_{t}}{G}=P_{0}-P_{L}-[P(0)-P(L)] .
$$

Substituting for $P(0)-P(L)$ in eq. (A3) above gives us (A5).

$$
J_{t}=\frac{(1+F) \alpha_{s} D_{w}\left[P_{0}-P_{L}\right]}{L+(1+F) 2 D_{w} \alpha_{s} / G}
$$

Let $\mathrm{V}$ be the volume flow rate in $\mathrm{cm}^{3} / \mathrm{sec}$ of the gas flushing the low $\mathrm{P}_{\mathrm{O}_{2}}$ chamber. Let $\mathrm{A}$ be the membrane area in $\mathrm{cm}^{2}$. Then if there is rapid, uniform mixing of gases and $V \gg J_{i}$.

$$
\mathrm{P}_{\mathrm{L}}=\mathrm{J}_{\mathrm{t}} \cdot 22.4 \frac{10^{3} \mathrm{~A}}{\mathrm{~V}} \quad 760=\mathrm{J}_{\mathrm{t}} \frac{\mathrm{A}}{\mathrm{V}} 17 \times 10^{6} \mathrm{~mm} \mathrm{Hg} .
$$

Substituting for $P_{L}$ in eq. (A5) and solving for $J_{t}$ gives eq. (A7).

$$
J_{t}=\frac{(1+F) D_{w} \alpha_{s} P_{0}}{L+(1+F) 2 D_{w} \alpha_{s}\left[\frac{1}{G}+\frac{A}{2 V}\left(17 \times 10^{6}\right)\right]} .
$$


For Wittenberg's experiments let us take $A=9 \mathrm{~cm}^{2}, \mathrm{~V}=1 \mathrm{~cm}^{3} / \mathrm{sec}$. Then $17 \times 10^{6} \mathrm{~A} /$ $2 \mathrm{~V}=1 /\left(1.3 \times 10^{-8}\right)$. Hence if $\mathrm{G}<1.3 \times 10^{-9}$ the small $\mathrm{P}_{\mathrm{L}}$ at the low $\mathrm{P}_{\mathrm{O}_{2}}$ side has little effect and $\mathrm{J}_{1}$ is given approximately by eq. (A8).

$$
J_{t} \simeq \frac{(1+F) D_{w} \alpha_{s} P_{0}}{L+(1+F) 2 D_{w} \alpha_{s} / G}
$$

However if $G>1.3 \times 10^{-7}$ the conductance term is negligible and the $P_{L}$ set by the flushing of the low $\mathrm{P}_{\mathrm{O}_{2}}$ side of the chamber plays the major role in eq. (A7) to give eq. (A9).

$$
J_{t} \simeq \frac{(1+F) D_{w} \alpha_{s} P_{0}}{L+(1+F) D_{w} \alpha_{s} A\left(1.7 \times 10^{7}\right) / V}
$$

\section{References}

Davies, J. T. and E. K. Rideal (1963). Interfacial Phenomena. New York, Academic Press.

Fatt, I. and R. C. LaForce (1961). Theory of oxygen transport through hemoglobin solutions. Science 133: 1919-1921.

Guastalla, J.(1939). Films très dilués de quelques protéines; essai de détermination des masses moléculaires. C. R. Acad. Sci. (Paris) 208: 1978-1080.

Jacquez, J. A. (1970). A First Course in Computing and Numerical Methods. Reading, Mass., AddisonWesley.

Keller, K. H. and S. K. Friedlander (1966). Diffusivity measurements of human methemoglobin. J. Gen. Physiol, 49: 681-687.

Kreuzer, F. (1970). Facilitated diffusion of oxygen and its possible significance; a review. Respir. Physiol. $9: 1-30$.

Kreuzer, F. and L. J. C. Hoofd (1970). Facilitated diffusion of oxygen in the presence of hemoglobin. Respir. Physiol. 8: 280-302.

Kutchai, H. (1970). Numerical study of oxygen uptake by layers of hemoglobin solution. Respir. Physiol. $10: 273-284$.

Kutchai, H., J. A. Jacquez and F. J. Mather (1970). Nonequilibrium facilitated oxygen transport in hemoglobin solution. Biophys. J. 10: 38-54.

Murray, J. D. (1968a). A simple method for obtaining approximate solutions for a class of diffusion-kinetics enzyme problems: I. General class and illustrative examples. Math. Biosc. 2: 379-411.

Murray, J. D. (1968b). A simple method for obtaining approximate solutions for a class of diffusion-kinetics enzyme problems: II. Further examples and nonsymmetric problems. Math. Biosc. 3: 115-133.

Murray, J. D. (1971). On the molecular mechanism of facilitated oxygen diffusion by haemoglobin and myoglobin. Proc. Roy. Soc. (London) B. 178: 95-110.

Sendroy, Jr. J., R. T. Dillon and D. D. van Slyke (1934). Studies of gas and electrolyte equilibria in blood. XIX The solubility and physical state of uncombined oxygen in blood. J. Biol. Chem. 105: 597-632.

Wittenberg, J. B. (1966). The molecular mechanism of hemoglobin-facilitated oxygen diffusion. $J$. Biol. Chem. 241 : 104-114.

Wittenberg, J. B. (1970). Myoglobin-facilitated oxygen diffusion: Role of myoglobin in oxygen entry into muscle. Physiol. Rev. 50: 559-636. 